\title{
Multi-field coupled color-changing devices and structures
}

\author{
BAO YinHua ${ }^{1}, \mathrm{HAN}_{\mathrm{Yu}}^{2}$, SONG WeiLi ${ }^{2}, \mathrm{CHEN} \mathrm{HaoSen}^{2} \&$ FANG DaiNing ${ }^{1,2 *}$ \\ ${ }^{1}$ State Key Laboratory for Turbulence and Complex System, College of Engineering, Peking University, Beijing 100871, China; \\ ${ }^{2}$ Institute of Advanced Structure Technology, Beijing Institute of Technology, Beijing 100081, China
}

Received December 21, 2018; accepted February 20, 2019; published online May 10, 2019

Citation: Bao Y H, Han Y, Song W L, et al. Multi-field coupled color-changing devices and structures. Sci China Tech Sci, 2019, 62: 1065-1066, https://doi. org/10.1007/s11431-018-9466-4

Multi-field coupling phenomenon plays a significant role in the design and fabrication of color-changing devices. By introducing external fields, such as strain/stress, electric, thermal, or chemical fields, these devices can be modulated for dynamic optical tunability. For instance, mechanochromism is the color change of a material induced by external force. By incorporating photonic crystals into polymer matrices, the resulting shape transformable materials enable to manipulate the flow of light upon mechanical strain stimulation [1]. Electrical field modulation has also been widely utilized in electrochromic materials, usually known as inorganic transition metal oxides and organic polymers. The electrochromic properties are based on the electric-field-induced intercalation/de-intercalation of small ions for transition metal oxides. While the evolution of some polymers electrochromism originates from the electrochemical doping/dedoping of the conjugated main chain. These materials can exhibit reversibly color change stimulated by redox reactions, depending on the applied potentials. Meanwhile, structural and materials designs have also been undertaken for altering their color upon exposure to multiple stimuli. Various nanostructures such as lamellar stacks, origami, and nanotips, are designed based on interference, reflection and antireflection $[2,3]$. By controlling their scales, these structures can be modulated for diverse optical functions with thin film interference and diffraction. For example, by controlling the layer thickness of lamellar stacks and optical parameters, the intensity of different wavelength

\footnotetext{
* Corresponding author (email: fangdn@pku.edu.cn)
}

will change simultaneously, showing various transmission spectrum or reflection spectrum. Especially, composite photonic gels show ternary (stress/strain, thermal, and $\mathrm{pH}$ ) stimuli-responsive behavior with the addition of thermal and pH sensitive materials into photonic matrices [4]. Material and structural designs combining with the multi-field coupling approaches have been the focus of intense research efforts and resulted in advances in the fabrication of smart windows, specular reflector, sensor, and biomimetic camouflage devices.

Recently, Daining Fang and co-workers [5] developed a prototype of bioinspired controllable electro-chemomechanical coloration films. This coloration film was fabricated by depositing a nanoscale amorphous silicon (a-Si) layer onto a reflective metal substrate. By the lithiation/delithiation of a-Si layer, such coloration films exhibited consecutively tunable chromogenic property in a broad visible band and provided a unique switch capability between chromatic and achromic reflectance spectrums under electro-chemomechanical control (Figure 1(a)). The fast color change property of the controllable coloration films was evaluated by the charge/discharge profiles under the ultrahigh current density of $40 \mu \mathrm{A} \mathrm{mm}^{-2}$ in well-chosen voltage range of $0.22-1.40 \mathrm{~V}$. The whole color-switching time was strictly divided into five representative color parts based on the standard Munsell color system. The switching time between two close representative colors was fast within a few seconds (Figure 1(b)). To illustrate the electro-chemomechanical mechanism in the coloration film, the spectroscopic ellipsometry models and simulation equivalent models were 

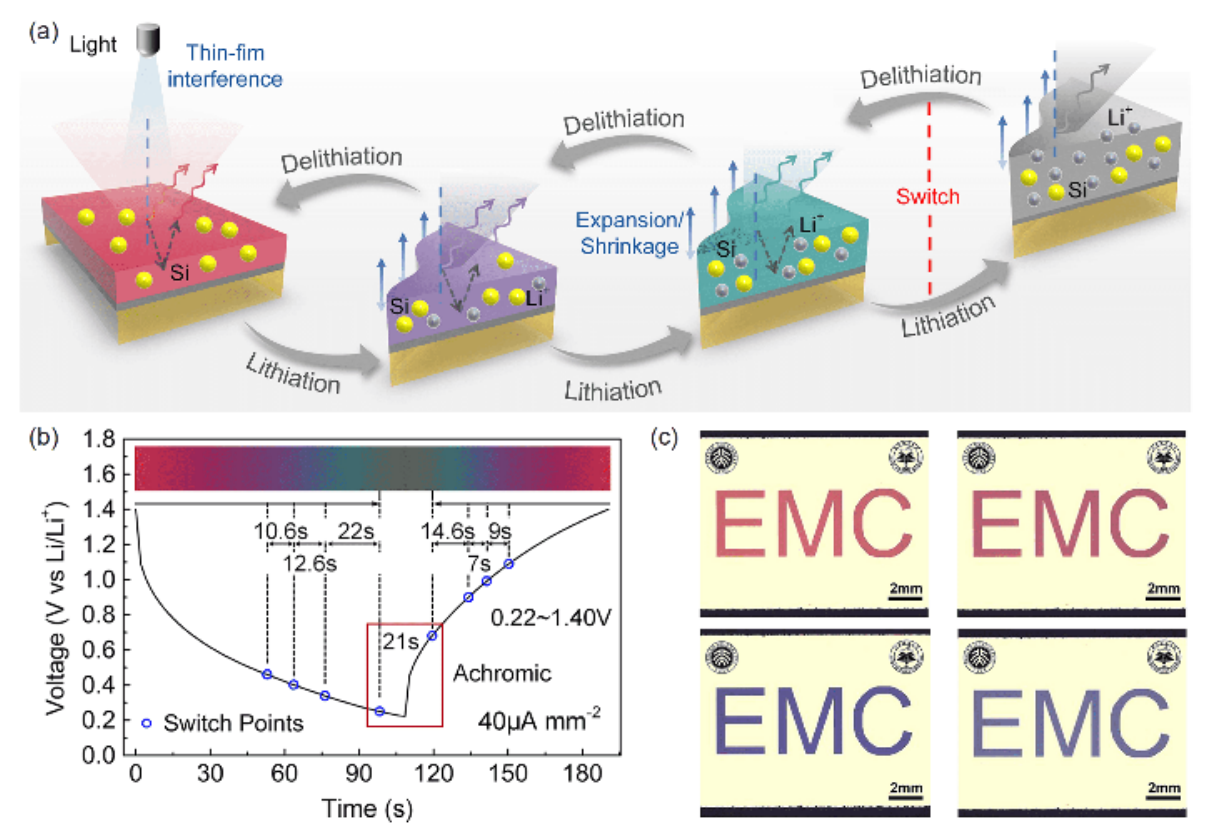

(c)

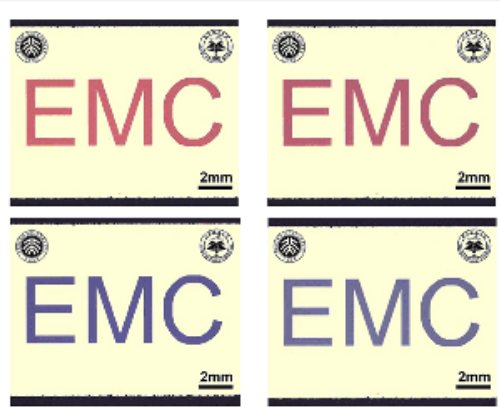

Figure 1 (Color online) (a) The chemical and physical coupled evolution of the modulating process of the controllable coloration films. (b) The fast charge/ discharge profiles for the coloration films. (c) The chromatic patterned silicon-based controllable coloration film. Reprinted with permission from ref. [5], Copyright 2019, Wiley-VCH.

established experimentally and theoretically. The nanoscaled a-Si film served as an enhancement reflection coating on a metalized film (copper). By insertion/extraction of Li-ion, the a-Si layer changed to $\mathrm{Li}_{x} \mathrm{Si}$ layer, leading to the optical component and thickness change of a-Si layer. This reversible chemical and physical coupled evolution contributed to the variable interference in the coloration films at different lithiated states. When the film was under high lithiated state, the high absorptivity and large thickness of the $\mathrm{Li}_{x} \mathrm{Si}$ film would weaken the interference effects, and finally, presented the achromic reflectance spectrum. Moreover, it had great designability via simply changing the thickness of initial a-Si nanofilms and the original color of reflective metal substrate. The coloration films can also be patterned into various shapes using the shadow mask technique (Figure 1(c)). It promises a novel multi-field coupled strategy for fabricating advanced coloration materials and devices that are pursued in sensors, labels, specular reflection, camouflage, and displaying.

1 Chan E P, Walish J J, Urbas A M, et al. Mechanochromic photonic gels. Adv Mater, 2013, 25: 3934-3947

2 Huang Y F, Chattopadhyay S, Jen Y J, et al. Improved broadband and quasi-omnidirectional anti-reflection properties with biomimetic silicon nanostructures. Nat Nanotech, 2007, 2: 770-774

3 Song Z, Lv C, Liang M, et al. Microscale silicon origami. Small, 2016, 12: 5401-5406

4 Yue Y F, Haque M A, Kurokawa T, et al. Lamellar hydrogels with high toughness and ternary tunable photonic stop-band. Adv Mater, 2013, 25: 3106-3110

5 Bao Y, Han Y, Yang L, et al. Bioinspired controllable electro-chemomechanical coloration films. Adv Funct Mater, 2019, 29: 1806383 